\section{Another rare facet of the ubiquitous mould}

Santosh Rai, ${ }^{1}$ Vinay Alva, ${ }^{2}$

Priya Pathak ${ }^{3}$ Vishak Acharya, ${ }^{4}$ Jane Mendonca ${ }^{4}$

${ }^{1}$ Department of Radiodiagnosis, Kasturba Medical College Mangalore, Manipal Academy of Higher Education (MAHE) Manipal; ${ }^{2}$ Consultant Surgeon Alva's Health Centre, Moodabidri; ${ }^{3}$ Department of Pathology, Kasturba Medical College Mangalore, Manipal Academy of Higher Education (MAHE), Manipal; ${ }^{4}$ Department of Respiratory Medicine, Kasturba Medical College Mangalore, Manipal Academy of Higher Education (MAHE), Manipal, India

\begin{abstract}
Pancoast syndrome is a commonly seen manifestation of lung malignancies, and benign causes are uncommon. We report a case of a patient without any known cause of immunosuppression who presented with Pancoast syndrome and a radiological mass like lesion in the lung apex, that was revealed be due to aspergillosis.
\end{abstract}

\section{Introduction}

Pancoast syndrome refers to an array of signs and symptoms caused by a lesion in the superior pulmonary sulcus. The features of this syndrome include pain and/or paraesthesia involving the ipsilateral upper limb, wasting of the intrinsic muscles of the hand, and Horner syndrome (which consists of ptosis, miosis, enophthalmos and anhidrosis). ${ }^{1}$ The syndrome is usually caused by a malignant apical lung tumour. Benign causes, although reported, are rare and vary widely with regard to their aetiology.

\section{Case report}

A 70-year-old male, non-smoker, presented to us with complaints of intermittent haemoptysis for the past one month and pain in the left shoulder and upper arm. The pain was described as severe, constant, and "shock-like" in nature. The patient had no known comorbidities and had no history of drug abuse, alcoholism or chronic steroid use. He had no history of tuberculosis, pneumonia or any chronic lung disease in the past. On physical examination, the vital signs were within normal limits and the respiratory system examination was unrevealing. Examination of the nervous system revealed features of Horner syndrome on the left side (ptosis, miosis, anhidrosis and enophthalmos) along with wasting of the thenar muscles of the left hand. The rest of the systemic examination was unremarkable. The routine blood reports were unremarkable as well, including glycated haemoglobin levels and HIV ELISA (Human Immunodeficiency Virus Enzyme Linked Immunosorbent Assay).

A chest radiograph was ordered and an inhomogeneous opacity was noted in the left apical region (Figure 1A, black arrow). With the clinical suspicion of Pancoast syndrome, a computed tomography scan of the chest was done which showed a minimally enhancing mass like lesion in the left lung apex (Figure 1B, black arrow) with hyperdense areas noted within the lesion which were presumed to be haemorrhage (Figure 1 $\mathrm{C}$ Black arrow). There were however no associated mediastinal nodes, secondary lung lesions, lung collapse, rib involvement or spiculations visualized in the wall of the lesion. The patient was advised to undergo flexible bronchoscopy for evaluation of the lesion and haemoptysis, but did not consent for the procedure. He agreed to undergo a percutaneous fine needle aspiration of the lesion, and this was done under CT guidance (Figure 1 D Black arrow). Cytological examination of the aspirate showed dense clusters of septate fungal organisms with occasional acute angle branching, suggestive of aspergillus (Figure 2A, B). A cell block prepared from the same sample also demonstrated the organisms which were GMS (Gomori methanamine silver) and PAS (Periodic acid-Schiff) positive (Figure 2C, D). Culture of the aspirate confirmed aspergillosis. A final diagnosis of primary pulmonary aspergilloma was made as there was no evidence of hypersensitivity (total and aspergillus specific serum IgE were within normal limits) or fungal tissue invasion. The patient was initiated on treatment with oral itraconazole but was later lost to physical follow up. He was, however, contacted telephonically 6 months later and reported resolution of symptoms.

\section{Discussion}

The eponymous Pancoast syndrome was first described by Henry Pancoast in $1924^{2}$ and is caused by a lesion located at the at the thoracic inlet, specifically in the apical pleuropulmonary groove adjacent to the subclavian artery and vein. The anatomy
Correspondence: Jane Mendonca, Department of Respiratory Medicine, Kasturba Medical College Mangalore, Manipal Academy of Higher Education (MAHE) Manipal.

Tel.: 91.08242422271

E-mail: enajmend516@gmail.com

Key words: Aspergillosis; fungal; Pancoast syndrome; tumour mimics.

Conflict of interest: The authors declare no conflict of interest.

Availability of data and materials: All data generated or analyzed during this study are included in this published article.

Informed consent: Written informed consent was obtained from a legally authorized representative(s) for anonymized patient information to be published in this article.

Received for publication: 2 May 2021.

Revision received: 24 January 2022.

Accepted for publication: 27 January 2022.

This work is licensed under a Creative Commons Attribution NonCommercial 4.0 License (CC BY-NC 4.0).

(C) Copyright: the Author(s), 2022

Licensee PAGEPress, Italy

Chest Disease Reports 2022; 9:9837

doi:10.4081/cdr.2022.9837

of the region places these lesions in close proximity to the brachial plexus, cervical sympathetic chain and stellate ganglion. The invasion of these structures is responsible for several classic clinical manifestations, including Horner syndrome. ${ }^{1}$ The commonest aetiology of Pancoast syndrome is a malignant lung tumour, so much so that the term 'Pancoast tumour' is synonymous with lung cancer in the definition provided by the American College of Chest Physicians (ACCP). ${ }^{3}$ Among the different histological subtypes of lung cancer responsible for Pancoast syndrome, non-small cell lung cancers constitute the vast majority. ${ }^{1}$ However, various other tumours have also been reported to cause Pancoast syndrome, including lymphoma, plasmacytoma, adenoid cystic carcinoma, mesothelioma, and metastases from other primary tumours (liver, cervix, bladder, thyroid). ${ }^{1}$ A benign tumour like solitary pleural fibroma has also been reported. ${ }^{4}$ Other benign causes of Pancoast syndrome that have been reported in the literature include vascular lesions and infections (bacterial and fungal). ${ }^{1,5}$

In a retrospective review of Pancoast syndrome secondary to infections, aspergillus was isolated as causative organism in 2 out of 31 cases. $^{6}$ Simpson et al. 
published a case of Pancoast syndrome caused by aspergillosis in a patient with acute myeloid leukaemia, as did Collins et al. The diagnosis was established in these cases using open lung biopsy and FNA, respectively. 7,8 Another case report documented Pancoast syndrome cause by chronic necrotizing aspergillosis in a patient with a personal history of bronchiectasis and breast carcinoma. ${ }^{9}$ Our case was uncommon in that aspergillus infection was seen in immunocompetent individual without chronic lung ailment. Aspergilloma is quite uncommon but has been reported in immunocompetent hosts. ${ }^{10}$ Long standing exposure to molds can still predispose immunocompetent individuals to develop aspergilloma.

There is little association with immunocompetent status of the individual with development of solid malignancy like lung cancers. However, haematological and lympho-proliferative malignancies have a predilection to develop in immunocompetent hosts.

Aspergillus in the lung can present with myriad of manifestations ranging from saprophytic colonization, hypersensitive reactions and semi-invasive disease, to invasive disease. All these four stages often evolve dynamically and clinical and radiological manifestations may fluctuate between these stages. In our case it is likely that initial colonization progressed to bronchial invasion by Aspergillus species and subsequent parenchymal cavitation with aspergilloma colonizing the cavity. ${ }^{11}$ The apical lesion in view of its anatomical location and proximity to the brachial plexus presumably led to compression of the brachial plexus as has been observed with superior sulcus tumours leading to Horner syndrome.

Although it is possible that there could have been a co-existing malignancy, this is rather unlikely. The patient was a nonsmoker and had no specific risk factors for malignancy. The CT features did not show any pathognomonic features of malignancy like rib erosion, spiculations, mediastinal nodes, collapse or associated satellite lung lesions. The FNAC and cell block analysis did not reveal any atypical cells or cells suspicious of malignancy either.

Similar to us, Das et al. reported a case of Pancoast tumour caused by pulmonary aspergillosis in an immunocompetent individual who presented with complaints similar to our patient. ${ }^{12}$ But contrary to our case, microbiological examination of the FNAC sample was negative and the authors had to proceed with a trucut biopsy of the lesion to arrive at the diagnosis. Guidelines recommend histopathological examination of lung tissue to make a diagnosis of pulmonary aspergillosis, due to the poor sensitivity of sputum and bronchial lavage specimens. ${ }^{13}$ However, this may not always be feasible, and FNAC could offer a cheaper, less invasive method of obtaining specimens for histopathological examination. ${ }^{14}$ Further studies are needed to establish the diagnostic yield of FNAC in pulmonary aspergillosis. One possible reason for immunocompetent individuals to have developed aspergillus infection is massive incidental

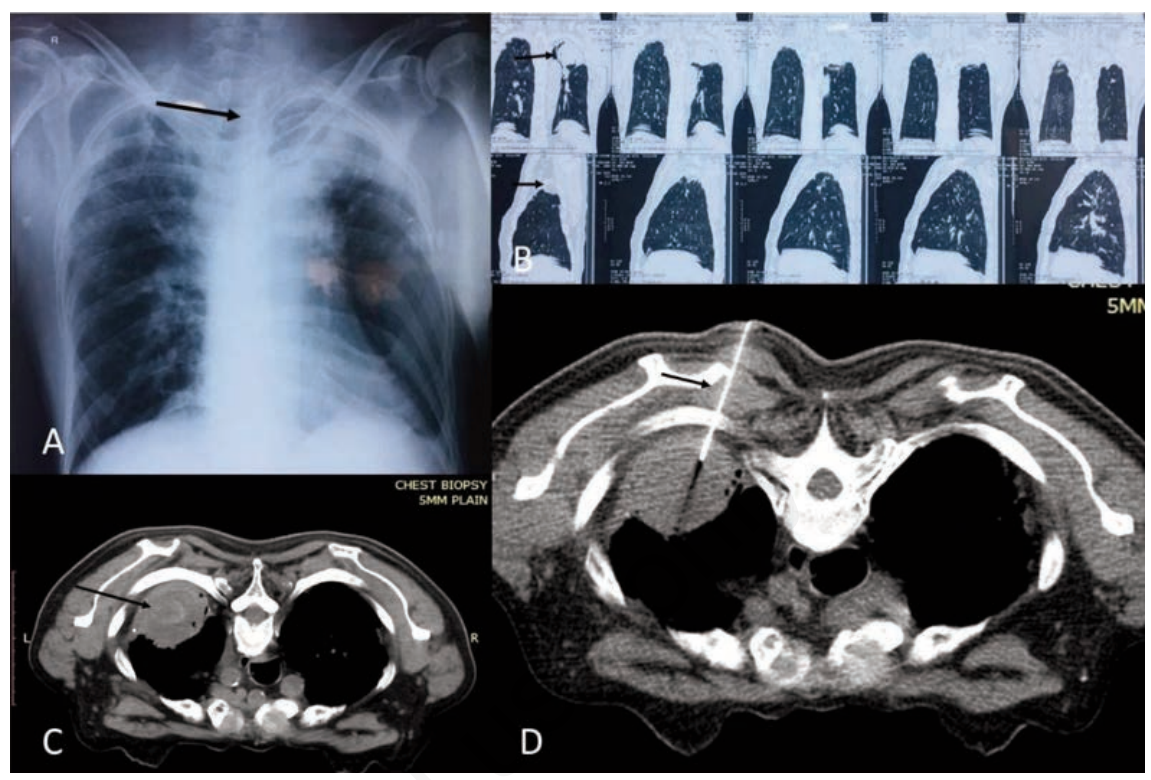

Figure 1. A: Chest radiography shows an ill-defined haziness in the left apical region (black arrow); B: CT Lung window Coronal reformats shows a soft tissue density with a minimally enhancing mass like lesion in the left apex (black arrow); C: Mediastinal window shows hyperdense areas within the lesion which were presumed to be haemorrhage (black arrow); and D: Mediastinal window shows the aspiration needle in the lesion during the sampling (black arrow).

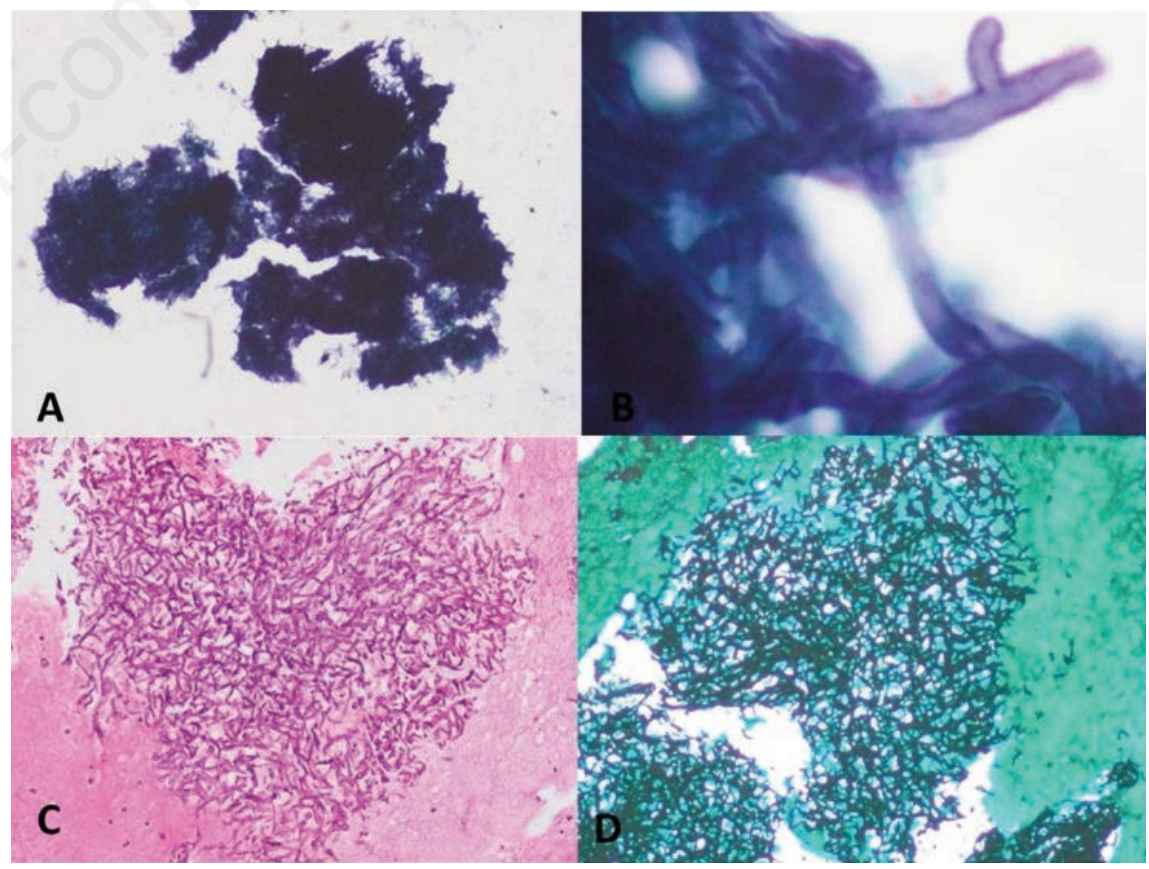

Figure 2. A: Thick clusters of fungal organisms seen in a clear background (PAP X 40); B: These were septate and branched at an acute angle (PAP X 1000); C, D: Cell block preparation showing positivity for fungal stains (PAS, GMS X 200). 
inoculation of aspergillus from the environment, as aspergillus is ubiquitous. Although our patient had no definite immunocompromised status, his advanced age could have been a risk factor for colonization and subsequent infection. Treatment options include oral itraconazole and intravenous amphotericin B, and complete resolution of the lesion can be seen with treatment. ${ }^{12}$

\section{References}

1. Panagopoulos N, Leivaditis V, Koletsis E, et al. Pancoast tumors: characteristics and preoperative assessment. J Thoracic Dis 2014;6:S108.

2. Pancoast H. Superior pulmonary sulcus tumor: tumor characterizedby pain, Horner's syndrome, destruction of bone andatrophy of hand muscles. JAMA 1932;99:1391-6.

3. Kozower BD, Larner JM, Detterbeck FC, Jones DR. Special treatment issues in non-small cell lung cancer. Diagnosis and management of lung cancer:
American College of Chest Physicians evidence-based clinical practice guidelines. Chest 2013;143:e369S-99S.

4. Fibla JJ, Penagos JC, León C. Pseudopancoast syndrome caused by a solitary fibrous tumor of the pleura. Archivos de bronconeumologia 2004;40:244-5.

5. Kwak Y. Vertebral artery dissection presenting as Pancoast's syndrome: A case report. Interdisciplinary Neurosurg 2021:101212.

6. White HD, Boethel C, Arroliga AC, White BA. Pancoast's syndrome secondary to infectious etiologies: a not so uncommon occurrence. Am J Med Sci 2011;341:333-6.

7. Simpson FG, Morgan M, Cooke NJ. Pancoast's syndrome associated with invasive aspergillosis. Thorax 1986;41:156-7.

8. Collins PW, Lord CD, Newland AC. Pancoast's tumour due to aspergilloma. Lancet (British edition). 1990;336(8730).

9. Ong TH, Eng P. Pancoast's syndrome due to chronic necrotizing pulmonary aspergillosis. Chest 2003;124:S317.
10. Clancy CJ, Nguyen MH. Acute community-acquired pneumonia due to Aspergillus in presumably immunocompetent hosts: clues for recognition of a rare but fatal disease. Chest 1998;114:629-34

11. Ofori A, Steinmetz AR, Akaasi J, et al. Pulmonary aspergilloma: An evasive disease. Int J Mycobacteriol 2016;5:235-9.

12. Das A, Choudhury S, Basuthakur S, et al. Pancoast's syndrome due to fungal abscess in the apex of lung in an immunocompetent individual: a case report and review of the literature. Case Rep Pulmonol 2014;2014.

13. Ullmann AJ, Aguado JM, ArikanAkdagli S, et al. Diagnosis and management of Aspergillus diseases: executive summary of the 2017 ESCMIDECMM-ERS guideline. Clinical Microbiol Infection 2018;24:e1-38.

14. Sharma S, Gupta P, Gupta N, et al. Pulmonary infections in immunocompromised patients: the role of image-guided fine needle aspiration cytology. Cytopathol 2017;28:46-54. 